\title{
Investigating the relationship between the severity of coronary artery disease and inflammatory factors of MHR, PHR, NHR, and IL-25
}

\author{
Hamed Manoochehri ${ }^{1,2+}$, Reza Gheitasi ${ }^{2,3+}$, Mona Pourjafar ${ }^{1,2+}$, Razieh Amini $^{1}$, Amirhossein Yazdi $^{4} *$ (D) \\ Received: 28 Jun 2020 \\ Published: 1 Jul 2021
}

\section{Abstract}

Background: Coronary artery disease (CAD), as a most common cause of death, is mainly caused by atherosclerosis. Due to the role of inflammation in the process of atherosclerosis, in the present study, the relationship between the severity of coronary artery disease and inflammatory factors of monocyte to HDL-C ratio (MHR), platelet-to-HDL-C ratio (PHR), neutrophil to HDL-C ratio (NHR), and IL-25 was investigated.

Methods: In this cross-sectional study, 64 patients with diagnosis of coronary artery disease who were undergoing angiography in Farshchian heart center in Hamadan were studied. For each patient, the count of monocytes, neutrophils, platelet, and HDL-C, and IL25 were measured from their blood and serum samples. Also, demographic information, such as age, gender, diabetes, smoking, and history of hypertension, was collected using a checklist. Data were described using frequency, percent, mean, and standard deviation. Statistical analysis was performed using independent $t$ test, Mann-Whitney, Wilcoxon, and Spearman rank correlation tests, and multiple linear regression by SPSS version 25.0 SPSS Inc). $\mathrm{P}<.05$ was considered as significant.

Results: The results of this study showed that IL-25 and MHR index has a significant correlation with coronary artery disease and Gensini score $(\mathrm{P}<.001)$. The PHR index was associated with coronary artery disease. Also, qualitative variables, such as history of hypertension, history of smoking, and gender, have a significant association with the severity of coronary artery disease $(\mathrm{P}<.05)$.

Conclusion: Among the inflammatory markers examined, IL-25 and MHR are stronger markers for assessing the severity of coronary artery disease. Simple and available IL-25 and MHR measurements may be able to, along with common risk factors and lipid profiles, predict the amount of vascular occlusion in treatment centers as an alternative of angiography as well as screening high risk patients prone to cardiovascular disease.

Keywords: Coronary Artery Disease, IL-25, HDL-C, MHR, NHR, PHR

Conflicts of Interest: None declared

Funding: Hamadan University of Medical Sciences (No. 9612087762)

\section{*This work has been published under CC BY-NC-SA 1.0 license.}

Copyright $\odot$ Iran University of Medical Sciences

Cite this article as: Manoochehri H, Gheitasi R, Pourjafar M, Amini R, Yazdi A. Investigating the relationship between the severity of coronary artery disease and inflammatory factors of MHR, PHR, NHR, and IL-25. Med J Islam Repub Iran. 2021 (1 Jul);35:85. https://doi.org/10.47176/mjiri.35.85

\section{Introduction}

Coronary artery disease (CAD) is the most common

Corresponding author: Dr Amirhossein Yazdi, amirhoseyn.yazdi@umsha.ac.ir

+ These authors have same contribution on this work.

1. Research Center for Molecular Medicine, Hamadan University of Medical Sciences, Hamadan, Iran

2. Student Research Committee, Hamadan University of Medical Sciences, Hamadan, Iran

3. Department of Immunology, School of Medicine, Hamadan University of Medical Sciences, Hamadan, Iran

4. Department of Cardiology, School of Medicine, Hamadan University of Medical Sciences, Hamadan, Iran

cause of death globally (17.9 million death each year) and

$\uparrow$ What is "already known" in this topic:

Atherosclerosis, buildup of plaque inside the artery walls, is an inflammatory disease and major cause of coronary artery disease (CAD). IL-25 (Interleukin-25), MHR (monocyte to high-density lipoprotein cholesterol ratio), NHR (neutrophil to high-density lipoprotein cholesterol ratio), and PHR (platelet to high density lipoprotein cholesterol ratio) are recently emerging inflammatory indicator.

\section{$\rightarrow$ What this article adds:}

MHR/IL-25/PHR were significantly associated with severity of coronary artery stenosis. MHR and IL-25 were 2 most reliable and powerful inflammatory markers for prediction of CAD severity. These markers have great potential for screening elective patients with stable symptoms. 
affects approximately 126 million individuals of the world's population $(1.72 \%$ or 1,655 per 100,000$)(1)$. Also, $\mathrm{CAD}$ is the first le ading cause of death in Iranian populations (262 death per 100,000, annually), with approximately an incidence of 12 per 1000 of population (2).

The majority of the CAD is related to atherosclerosis where the progressive occurrence of endothelial dysfunction, vascular inflammation, and thrombosis result in plaque formation and may cause lethal consequence, including acute myocardial infarction (AMI) and unexpected cardiac death. Due to silent atherosclerotic plaque progression, the death rate is high for cardiovascular diseases worldwide. Hence, attempts focus on identification of reliable novel biomarkers to predict the plaque characterization, formation, and diagnosis in early stages and timely treatment, subsequently declining morbidity and mortality of cardiovascular diseases (3).

Inflammation could be as one of the risk factors that contribute to the initiation and progression of atherosclerotic process by promoting plaque growth (4). Interlukin25 (IL-25 or IL-17E) is related to IL-17 cytokine family and is involved in the induction of T helper 2 (Th2) type immune responses (type 2 immune response) through production of the T helper 2 cytokines, such as IL-13, IL-5, and IL-4 (5). In addition, IL-25 can also prevent the immune responses mediated by Th1/Th17 (6). It has been demonstrated that mature B cells and also endothelial cells and smooth muscle cells (SMCs) of nor$\mathrm{mal} /$ atherosclerotic human arteries in progressive plaque constitutively express IL-25 (7). Furthermore, cytokines of $\mathrm{T}$ helper 2 may play a role in local inflammatory response, because they are present in the plaque (5). However, the researches on the role of IL-25 in CAD (protective or plaque stabilizer) is limited and challenging $(8,9)$.

High-density lipoprotein cholesterol (HDL-C) is a fundamental groups of human lipoproteins that has antiatherogenic properties and reverse relationship with CAD through inhibition of macrophages migration, reduction of low-density lipoprotein cholesterol (LDL-C) oxidation, promotion of cholesterol flow from these cells, and prevention of platelet aggregation $(10,11)$. On the other hand, blood components such as platelets and circulating white blood cells (WBCs), including monocytes, lymphocytes, eosinophils, and neutrophils, are involved in atherosclerosis inflammatory processes. As known, circulating monocytes interact with endothelial cells and platelets via secretion of different factors and cytokines, and subsequently initiating inflammatory and prothrombotic pathways (12). It has been reported that a recently emerged inflammation-based marker, the monocytes to HDL-C ratio (MHR), could reflect the inflammatory status of a patient and potentially can be used as a novel prognostic and predictor factor for cardiovascular diseases $(13,14)$. However, platelet to lymphocyte ratio (PLR) and neutrophil to lymphocyte ratio (NLR) has been indicated to be inversely associated with HDL in CAD patients $(10,15)$.

At the moment, angiography is the gold standard for diagnosing coronary artery disease. Despite its high diagnostic accuracy, angiography has drawbacks and limitations, including aggressiveness, the use of ionizing radia- tion, high cost, and the need for special conditions and facilities. Therefore, medical researchers are trying to offer new noninvasive methods for diagnosing coronary artery disease that do not have the mentioned limitations. The above-mentioned markers can potentially provide simple, available, effective, and cheap tools for the prognosis and diagnosis of CAD even in the asymptomatic patients. However, no data are available on the association of MHR, neutrophil count to HDL-C ratio (NHR), platelet count to HDL-C ratio (PHR) inflammatory parameters, and IL-25 with the severity of CAD and atherosclerosis. Thus, in this study, we aimed to investigate these relations to predict coronary artery disease and their potential to be noninvasive diagnostic biomarkers for assessment of severity of coronary artery disease and prognosis.

\section{Methods}

\section{Patients and Samples}

In this cross-sectional study, patients with an initial diagnosis of coronary artery disease who were undergoing angiography, including 43 males and 21 females, and with a mean age of $55.3 \pm 7.8$ years who referred to the heart hospital of Hamedan University of Medical Sciences between January 2018 and July 2019 were recruited for this study according to the strict inclusion criteria. Based on the reported correlation coefficient $(\mathrm{r}=0.407)$ between MHR and Gensini score (12) and considering the $95 \%$ confidence level and test power of $90 \%$, the calculated minimum required sample size was approximately 59 patients using the following formula. However, 64 patients were enrolled in study considering about $10 \%$ probability of sample loss.

$$
n=\left(\frac{z_{1-\frac{\alpha}{2}}+z_{1-\beta}}{0.5 \ln \left(\frac{1+r}{1-r}\right)}\right)^{2}+3
$$

Elective patients (nonemergency patients) with stable symptoms were selected based on clinical evidences of coronary artery involvement. Patients with serious heart failure (eg, congenital heart disease, acute infarction, acute coronary syndrome), rheumatic disease, coagulation diseases kidney failure, infectious diseases, connective tissue diseases, malignancies, diabetes and consumption of immunosuppressive drugs were excluded from the study. After a detailed physical examination, the demographic data were recorded. Angiographic data were measured and recorded by an expert cardiologist. Patients were categorized into 4 groups according to coronary angiography, including patients without stenosis or with less than $50 \%$ stenosis in one of the coronary arteries (group 1, $\mathrm{n}=16$ ), patients with $50 \%$ or more stenosis in one of the coronary arteries (group 2, $\mathrm{n}=16$ ), patients with more than $50 \%$ stenosis in 2 coronary arteries (group $3, n=16$ ), patients with more than $50 \%$ stenosis in 3 coronary arteries (group 4, $\mathrm{n}=16$ ). They were scored through the Gensini score. Peripheral blood samples of patients were collecting before angiography for the following experiments. All patients were enrolled after providing informed consents. 
This study was approved by the research ethics committee, Hamadan University of Medical Sciences (Ethics code: IR.UMSHA.REC.1396.833).

\section{Biochemical and Hematological Parameters}

An automated hematology analyzer (Sysmex Kx-21NL) was used for measuring the total and differential leukocyte counts. Absolute cell counts were confirmed with the smear analyses. A biochemical autoanalyzer (BT 3000, auto-analyzer) was used for high-density lipoprotein cholesterol (HDL-C) measurement. MHR/PHR/NHR were computed as monocyte/platelet/neutrophil counts divided by HDL-C amount. IL-25 levels were measured by separating serum samples from peripheral blood of patients and using ELISA method according to manufacturer's protocol (Eastbiopharm Co).

\section{Statistical Analysis}

Quantitative and qualitative variables are defined as the mean \pm standard deviation (SD) and frequency (percent), respectively. Independent t test, Mann-Whitney, and Wilcoxon tests were used for comparing the mean/mean ranks of quantitative variables between 2 or more groups. Correlations between quantitative variables were assessed using Spearman rank correlation test. Multiple linear regression analysis was used to predict CAD severity according to independent variables. $\mathrm{P}<.05$ was considered as significant. All statistical analyses were performed using SPSS version 25.0 (SPSS Inc).

\section{Results}

A total of 64 patients with the diagnosis of CAD were enrolled. Patients were classified according to their hypertension, family history of coronary artery disease, and smoking. The demographic, clinical, and laboratory data of the patients are given in Table 1.

Spearman correlation test was used to investigate the relationship between NHR, MHR, PHR, and IL-25 serum levels with the Gensini score. Data analysis results showed a significant positive correlation between MHR $(\mathrm{r}=0.543, \mathrm{p}<0.001)$, PHR $(\mathrm{r}=0.374 ; \mathrm{p}=0.002)$, with the Gensini score and negative correlation between IL-25 serum level $(r=-0.307 ; p=0.014)$, with the Gensini score (Table 2).

The mean of NHR, MHR, PHR, and IL-25 serum levels were compared between different severity of coronary artery disease using Kruskal-Wallis statistical test (Table 3). Data analysis results revealed that increase of MHR $(p<0.001)$, PHR $(p=0.008)$ and decrease of IL-25 $(p=0.009)$ were significant in the groups with different severity of coronary artery disease. There was no significant difference in the mean of NHR between groups $(\mathrm{p}=0.456)$.

The Mann-Whitney test was used to compare the mean rank of PHR/MHR/NHR/IL-25 between the 2 levels of explanatory variables of hypertension, family history of coronary artery disease, smoking, and gender. Results of statistical analysis showed that NHR mean was significantly different between males and females $(p=0.037)$. Also, MHR mean was significantly different between patients with and without hypertension $(p=0.020)$ and between smokers and nonsmoker patients $(p=0.033)$. PHR mean was significantly different between males and females $(p=0.025)$, between patients with and without hypertension $(\mathrm{p}=0.019)$, and between smokers and nonsmoker patients $(\mathrm{p}=0.033)$.

Independent $\mathrm{t}$ test was used to compare the Gensini score mean between the 2 levels of explanatory variables of hypertension, family history of coronary artery disease, smoking, and gender. There was a significant difference in the mean of Gensini score between males and females $(p=0.001)$, between patients with and without hypertension $(\mathrm{p}=0.013)$, and between smokers and nonsmoker patients $(\mathrm{p}=0.033)$

Spearman correlation test was used to investigate the relationship between PHR/MHR/ NHR/IL-25/Gensini score and age .Results showed that only the Gensini score was significantly correlated with age $(r=0.294 ; p=0.019)$ (Table 4).

Multiple linear regression was used to predict the Gen-

Table 1. Clinical and laboratory characteristics

\begin{tabular}{|c|c|c|}
\hline Parameters & \multicolumn{2}{|c|}{ Total $(\mathrm{N}=64)$} \\
\hline Age (years) & \multicolumn{2}{|c|}{$55.3 \pm 7.8$} \\
\hline Male sex $[\mathrm{n}(\%)]$ & \multicolumn{2}{|c|}{$43[67.2 \%]$} \\
\hline Female sex $[\mathrm{n}(\%)]$ & \multicolumn{2}{|c|}{$21[32.8 \%]$} \\
\hline Smoking $[\mathrm{n}(\%)]$ & \multicolumn{2}{|c|}{$13[20.3 \%]$} \\
\hline Hypertension [n (\%)] & \multicolumn{2}{|c|}{$22[34.4 \%]$} \\
\hline Family history of CAD $[\mathrm{n}(\%)]$ & \multicolumn{2}{|c|}{$11[17.2 \%]$} \\
\hline NHR [count.dl/mg] & \multicolumn{2}{|c|}{$2.52 \pm 1.3$} \\
\hline PHR [count.dl/mg] & \multicolumn{2}{|c|}{$2.52 \pm 1.3$} \\
\hline MHR [count.dl/mg] & \multicolumn{2}{|c|}{$0.15 \pm 0.1$} \\
\hline IL-25 [pg/ml] & \multicolumn{2}{|c|}{$24.87 \pm 3.76$} \\
\hline Gensini score & \multicolumn{2}{|c|}{$45.37 \pm 1.3$} \\
\hline \multicolumn{3}{|c|}{ Correlation with Gensini score } \\
\hline Variable & Spearmen Correlation (R) & $\mathrm{p}$ \\
\hline NHR & 0.207 & 0.101 \\
\hline MHR & 0.543 & $<0.001^{*}$ \\
\hline PHR & 0.374 & $0.002^{*}$ \\
\hline IL-25 & -0.307 & $0.014^{*}$ \\
\hline
\end{tabular}


Table 3. The relationship between inflammatory factors and the severity of coronary artery disease

\begin{tabular}{lccccc}
\hline Variable & Group 1 & Group 2 & Group 3 & $\begin{array}{c}\text { Group 4 } \\
\text { P-Value }\end{array}$ & \\
\hline NHR (count.dl/mg) & $2.16 \pm 0.56$ & $2.86 \pm 2.23$ & $2.33 \pm 0.55$ & $2.73 \pm 1.07$ & 0.456 \\
MHR (count.dl/mg) & $0.08 \pm 0.05$ & $0.14 \pm 0.12$ & $0.14 \pm 0.06$ & $0.22 \pm 0.11$ \\
PHR (count.dl/mg) & $7.30 \pm 1.89$ & $9.95 \pm 7.34$ & $7.58 \pm 1.51$ & $11.50 \pm 4.83$ & $<0.001^{*}$ \\
IL-25 (pg/ml) & $26.51 \pm 4.15$ & $24.94 \pm 3.31$ & $25.64 \pm 3.32$ & $22.37 \pm 3.19$ & $0.008^{*}$ \\
\hline
\end{tabular}

Table 4. Comparison of NHR/MHR/PHR/IL-25/Gensini score mean between the two levels of qualitative variables and their relationship with age.

\begin{tabular}{|c|c|c|c|c|c|c|c|c|c|c|c|}
\hline \multirow[t]{2}{*}{ Variable } & & \multicolumn{2}{|c|}{$\begin{array}{c}\text { NHR } \\
\text { count.dl/mg }\end{array}$} & \multicolumn{2}{|c|}{$\begin{array}{c}\text { PHR } \\
\text { count.dl/mg }\end{array}$} & \multicolumn{2}{|c|}{$\begin{array}{c}\text { MHR } \\
\text { count.dl/mg }\end{array}$} & \multicolumn{2}{|c|}{$\begin{array}{l}\text { IL-25 } \\
\mathrm{pg} / \mathrm{ml}\end{array}$} & \multicolumn{2}{|c|}{ Gensini score } \\
\hline & & Mean \pm SD/R & $\mathrm{p}$ & Mean \pm SD/R & $\mathrm{p}$ & Mean \pm SD/R & $\mathrm{p}$ & Mean \pm SD/R & $\mathrm{p}$ & Mean \pm SD/R & $\mathrm{p}$ \\
\hline \multirow{2}{*}{ Hypertension } & Yes & $2.61 \pm 0.96$ & 0.110 & $10.04 \pm 4.48$ & $0.019^{*}$ & $0.18 \pm 0.11$ & $0.020^{*}$ & $24.97 \pm 3.94$ & 0.832 & $2.78 \pm 1.06$ & $0.013 *$ \\
\hline & No & $2.47 \pm 1.45$ & & $8.58 \pm 4.91$ & & $0.13 \pm 0.09$ & & $24.81 \pm 3.71$ & & $1.87 \pm 1.32$ & \\
\hline \multirow{4}{*}{$\begin{array}{l}\text { Family histo- } \\
\text { ry of CAD } \\
\text { Smoking }\end{array}$} & Yes & $2.39 \pm 0.55$ & 0.866 & $9.48 \pm 3.29$ & 0.505 & $0.15 \pm 0.17$ & 0.408 & $23.70 \pm 3.04$ & 0.251 & $2.79 \pm 1.14$ & 0.137 \\
\hline & No & $2.5 \pm 1.41$ & & $9.0 \pm 5.05$ & & $0.15 \pm 0.11$ & & $25.11 \pm 3.88$ & & $2.04 \pm 1.31$ & \\
\hline & Yes & $3.44 \pm 1.41$ & $0.063^{*}$ & $12.77 \pm 8.55$ & $0.033^{*}$ & $0.23 \pm 016$ & $0.033^{*}$ & $24.82 \pm 4.21$ & 0.960 & $2.94 \pm 1.09$ & $0.033 *$ \\
\hline & No & $2.29 \pm 0.66$ & & $8.14 \pm 2.61$ & & $0.13 \pm 0.07$ & & $24.88 \pm 3.68$ & & $1.99 \pm 1.28$ & \\
\hline \multirow[t]{2}{*}{ Gender } & Male & $2.68 \pm 1.47$ & $0.037^{*}$ & $9.74 \pm 5.42$ & $0.025^{*}$ & $0.16 \pm 0.11$ & 0.287 & $24.56 \pm 3.75$ & 0.320 & $2.56 \pm 1.14$ & $0.001 *$ \\
\hline & Female & $2.2 \pm 0.78$ & & $7.74 \pm 2.72$ & & $0.12 \pm 0.05$ & & $25.49 \pm 3.12$ & & $1.42 \pm 1.29$ & \\
\hline Age & & 0.091 & 0.475 & 0.141 & 0.266 & 0.036 & 0.779 & -0.056 & 0.661 & 0.294 & $0.019^{*}$ \\
\hline
\end{tabular}

Table 5. Regression coefficients for Gensini score

\begin{tabular}{|c|c|c|c|c|c|}
\hline \multirow[b]{2}{*}{ Variable } & \multicolumn{2}{|c|}{ Unstandardized coefficients } & \multirow[b]{2}{*}{ Standardized coefficients Beta } & \multirow[b]{2}{*}{$\mathrm{t}$} & \multirow[b]{2}{*}{$\mathrm{p}$} \\
\hline & $\mathrm{B}$ & Std. error & & & \\
\hline (Constant) & -12.00 & 54.477 & - & -0.220 & 0.826 \\
\hline Age & 1.37 & 0.498 & 0.281 & 2.75 & 0.008 * \\
\hline IL-25 & -1.91 & 1.02 & -0.198 & -1.87 & 0.067 \\
\hline PHR & 3.07 & 2.70 & 0.350 & 1.13 & 0.262 \\
\hline NHR & -22.64 & 8.69 & -0.702 & -2.60 & $0.012 *$ \\
\hline MHR & 230.96 & 56.42 & 0.696 & 4.09 & $0.000 *$ \\
\hline Gender (ref $=$ male $)$ & -19.76 & 8.89 & -0.245 & -2.22 & 0.031 * \\
\hline Hypertension (ref=yes) & -2.83 & 8.52 & -0.036 & -0.333 & 0.741 \\
\hline Family history of CAD (ref=yes) & -6.27 & 10.91 & -0.062 & -0.575 & 0.568 \\
\hline Smoking (ref=yes) & -15.10 & 11.69 & -0.160 & -1.29 & 0.202 \\
\hline
\end{tabular}

sini score using independent variables (NHR, MHR, PHR, serum level of IL-25, age, gender, hypertension, and family history of CAD and smoking). The statistical model for quantitative variables has a $\mathrm{P}$ value less than .001 $(\mathrm{p}<0.001)$ and adjusted $\mathrm{R}^{2}$ of 0.398 . The variables, NHR $($ Beta $=-0.702 ; \mathrm{p}=0.012)$, MHR $($ Beta $=0.696, p<0.001)$, age $(\mathrm{Beta}=0.281 ; \mathrm{P}=.008)$, and gender $($ Beta $=-0.245$; $\mathrm{p}=0.031$ ) can determine the Gensini score. According to standardized coefficients beta, it is concluded that the NHR and MHR are the most important and determinant variable in this regard (Table 5).

\section{Discussion}

Cardiovascular disease has become the most cause of death in global societies. CAD is a widespread heart disease, which is more common in advanced societies and in older people. Since current treatments for CAD are mostly palliative, early detection is more important to prevent disease progression and mortality over the coming years. Hence, introducing novel reliable biomarkers for early detection and prediction of atherosclerosis as a major cause of CAD is required (16-18). HDL, a high-density lipoprotein, is present in the bloodstream and prevents the accumulation of free cholesterol and triglycerides in the vessels and formation of lipid plaques. Monocytes neutrophil and platelets, as inflammatory immune system components, are active in the bloodstream and play an important and essential roles in inflammation through secre- tion protein substances such as cytokines $(12,19)$. However, there are limited studies regarding the relationship between inflammatory/anti-inflammatory cytokines and coronary artery stenosis severity in CAD patients.with

the Relationship the severity of coronary artery disease and inflammatory factors of

The aim of the present study was to find the association between CAD severity with MHR, PHR, NHR (inflammatory markers), and IL-25 (anti-inflammatory cytokine).

We observed a significant correlation between the Gensini score with MHR and PHR indicators and also serum levels of IL-25. In the regression model, NHR, MHR, PHR and serum levels of IL-25 were considered as independent variables for prediction and age of the Gensini score in patients. The analysis showed that MHR index is the strongest predictor in this regard.

In present study, we showed that IL-25 serum level has been declined in CAD patients by progression of severity. Patients with more than $50 \%$ stenosis in 3 coronary arteries had the higher level of IL-25. Expression of IL-25 via resident plaque cells and presence of IL-25 positive B cells in progressive plaques were found in patients with atherosclerosis (7). Mantani et al indicated that IL-25 has an atheroprotective role in the apolipoprotein (apo) E deficient mice aortic arch via increasing Th1/Th17-mediated immune responses (9), and also they previously showed that the protective role of IL-25 is due to induction of IL5 , natural antiphosphorylcholine antibody generation by 
B1a cells, and expansion of innate lymphoid type 2 cells (ILC2) in atherosclerosis-prone apo-E deficient mice (5). On the other hand, Xu et al reported that IL-25 was significantly higher in coronary arteries of CAD patients when compared with normal coronary arteries, especially in acute myocardial infarction (AMI), and IL-25 was positively corelated with the Gensini score and TNF- $\alpha$ and IL6 levels $(\mathrm{r}=0.495 ; \mathrm{p}<0.001)$ in $\mathrm{CAD}$ patients. Based on their data, macrophages and $\mathrm{T}$ lymphocyte are important sources of IL-25 in human atherosclerotic plaques (8), while our data showed that IL-25 is negatively correlated with the Gensini score and there was no relationship between IL-25 expression and risk factor of CAD.

Furthermore, we have demonstrated the association of MHR/NHR/PHR with the CAD severity alone and in the context of the Gensini score. Our results showed that only MHR and PHR are linked to the Gensini score and CAD severity, so that these inflammation biomarkers are elevated in patients with stenosis in more coronary arteries.

Monocyte migrate into the subendothelial space and uptake lipoproteins and their migration is considerably more in hypercholesterolemic conditions. It seems that monocytosis during the $\mathrm{CAD}$ and $\mathrm{AMI}$ is contributed with plaque progression and high monocyte counts could be an independent marker for CAD and AMI (20). On the other hand, the HDL-C molecule have anti-atherosclerotic properties as a result of a phenomenon named "reverse cholesterol transport" and the HDL-C molecule prevents monocyte activation and recruiting (21). Consistent with our result, in Kundi's study, MHR was significantly higher in patients with high SYNTAX scores and potentially can be considered as a prognostic factor in stable CAD patients, with sensitivity of $66 \%$ and a specificity of $65.1 \%$ (13). Also, Cetin et al reported that MHR can be a predicator of CAD severity and it is possible to use MHR for identify high-risk patients for major adverse cardiovascular events. In their study, MHR had a significant and positive correlation with CRP, neutrophil to lymphocyte ratio, and SYNTAX, and the Gensini score (12).

Platelets has an important role in the etiology of CAD, and larger MPV (mean platelet volume) is associated with CAD (22). During atherosclerosis, a clot forms in an intact blood vessel that promotes platelet aggregates, and subsequently causing a blockage of blood flow and heart attack. In addition to this, HDL exhibits antithrombotic activity via prohibition of platelet aggregation (23). It has been previously mentioned that platelet-lymphocyte ratio (PLR) is inversely associated with HDL in CAD, and CAD patients having low HDL levels have higher PLR (10). In addition, PLR correlates with the higher CAD severity and poor prognosis in Chinese population, so it can be used as a predictive biomarker of CVD and risk of future CVD events (24). Although the role of platelets in pathogenesis of CAD is well known, the documented relationship between PHR and the Gensini score and intensity of CAD and our data are shown here for first time.

Neutrophils are highly efficient effector cells, shortlived, rather crude, and unrefined phagocytes that mobilize and spread to sites of inflammation. Also, they act as a main protagonist in the first line of immunity under acute inflammatory conditions. Neutrophils has been rarely detected in atherosclerotic lesions compared to other immune cells, and their contribution was largely unknown $(25,26)$. Kalay et al found that circulating neutrophil count and NLR were related with progression of atherosclerosis. Patients with high NLR showed higher progression rate (27). Nilsson reported an enhancement of neutrophil count in noncalcified plaques from patients with CAD, although this association was not seen for total plaque burden (28). Chen et al demonstrated mild positive correlations between the NLR and proportions of neutrophils with the Gensini score and suggest the NLR as an independent predictor of the severity of CAD assessed according to the Gensini score (29). Furthermore, according to a study by Zhang et al, NLR of 2.385 or higher predicted high Gensini scores, with a sensitivity of $64 \%$ and specificity of $63 \%$ (30). However, our result did not show the correlation between NHR, CAD severity, and Gensini scores.

Finally, in our study, IL-25/MHR/PHR (especially IL25 and MHR) were identified as powerful and reliable markers for diagnosing and estimating the severity of coronary artery stenosis. Also, these markers can potentially be used for disease monitoring and evaluation of response to treatment. However, this hypothesis requires further researches in this regard.

\section{Conclusion}

Among the examined inflammatory markers, MHR and IL-25 were more reliable and powerful for prediction of Gensini scores and CAD severity. Thus, these markers are potentially able to be used in daily clinical practice as an inexpensive and quickly accessible marker for screening elective patients with stable symptoms as an alternative of costly and invasive angiography procedure. We suggest that performances of these markers be investigated in different aspects of CAD disease, including disease progression and monitoring in future studies.

\section{Acknowledgement}

This study was supported by Hamadan University of Medical Sciences (No. 9612087762). We would like to thank our colleagues in Farshchian Heart Center, Hamadan University of Medical Sciences for their encouragement.

\section{Conflict of Interests}

The authors declare that they have no competing interests.

\section{References}

1. Khan MA, Hashim MJ, Mustafa H, Baniyas MY, Al Suwaidi SKBM, AlKatheeri R, et al. Global Epidemiology of Ischemic Heart Disease: Results from the Global Burden of Disease Study. Cureus. 2020;12(7):9349.

2. Sarrafzadegan N, Mohammmadifard N. Cardiovascular disease in Iran in the last 40 years: prevalence, mortality, morbidity, challenges and strategies for cardiovascular prevention. Arch Iran Med. 2019;22(4):204-10.

3. Otsuka F, Yasuda S, Noguchi T, Ishibashi-Ueda H, therapy. Pathology of coronary atherosclerosis and thrombosis. Cardiovasc Diagn Ther. 


\section{6;6(4):396-408.}

4. Kharazmi-Khorassani J, Asoodeh A, Tanzadehpanah H. Antioxidant and angiotensin-converting enzyme (ACE) inhibitory activity of thymosin alpha-1 (Tha1) peptide. Bioorg Chem. 2019;87(June 2019):743-52.

5. Mantani PT, Dunér P, Bengtsson E, Alm R, Ljungcrantz I, Söderberg I, et al. IL-25 inhibits atherosclerosis development in apolipoprotein E deficient mice. PloS One. 2015;10(1):0117255.

6. Liu Y, Shao Z, Shangguan G, Bie Q, Zhang B. Biological Properties and the Role of IL-25 in Disease Pathogenesis. J Immunol Res. 2018;2018(6519465):1-8.

7. de Boer OJ, van der Meer JJ, Teeling P, van der Loos CM, Idu MM, van Maldegem F, et al. Differential expression of interleukin-17 family cytokines in intact and complicated human atherosclerotic plaques. J Pathol. 2010;220(4):499-508.

8. Xu Y, Ye J, Wang M, Liu J, Wang Z, Jiang H, et al. The expression of interleukin-25 increases in human coronary artery disease and is associated with the severity of coronary stenosis. Anatol J Cardiol. 2020;23(3):151-9.

9. Mantani PT, Dunér P, Bengtsson E, Ljungcrantz I, Sundius L, To F, et al. Interleukin-25 (IL-25) has a protective role in atherosclerosis development in the aortic arch in mice. J Biol Chem. 2018;293(18):6791-801.

10. Prajapati JH, Sahoo S, Nikam T, Shah KH, Maheriya B, Parmar M. Association of high density lipoprotein with platelet to lymphocyte and neutrophil to lymphocyte ratios in coronary artery disease patients. J Lipids. 2014;2014:686791.

11. Wu T-T, Zheng Y-Y, Chen Y, Yu Z-X, Ma Y-T, Xie X, et al. Monocyte to high-density lipoprotein cholesterol ratio as long-term prognostic marker in patients with coronary artery disease undergoing percutaneous coronary intervention. Lipids Health Dis. 2019;18(1):180.

12. Cetin MS, Cetin EHO, Kalender E, Aydin S, Topaloglu S, Kisacik $\mathrm{HL}$, et al. Monocyte to HDL cholesterol ratio predicts coronary artery disease severity and future major cardiovascular adverse events in acute coronary syndrome. Heart Lung Circ. 2016;25(11):1077-86.

13. Kundi H, Kiziltunc E, Cetin M, Cicekcioglu H, Cetin ZG, Cicek G, et al. Association of monocyte/HDL-C ratio with SYNTAX scores in patients with stable coronary artery disease. Herz. 2016;41(6):523-9.

14. Korkmaz A, Demir M, Unal S, Yildiz A, Ozyazgan B, Demirtas B, et al. Monocyte-to-high density lipoprotein ratio (MHR) can predict the significance of angiographically intermediate coronary lesions. Int J Cardiovasc Acad. 2017;3(1-2):16-20.

15. Yüksel M, Yıldız A, Oylumlu M, Akyüz A, Aydın M, Kaya H, et al. The association between platelet/lymphocyte ratio and coronary artery disease severity. Anatol J Cardiol. 2016;15(8):640-7.

16. Lubrano V, Balzan S. Consolidated and emerging inflammatory markers in coronary artery disease. World journal of experimental medicine. World J Exp Med. 2015;5(1):21-32.

17. Manoochehri H, Sheykhhasan M, Samadi P, Pourjafar M, Saidijam M. System biological and experimental validation of miRNAs target genes involved in colorectal cancer radiation response. Gene Rep. 2019; $17: 100540$.

18. Gheitasi R, Jourghasemi S, Pakzad I, Sarmadi VH, Samieipour Y, Sekawi Z, et al. A potential marker in brucellosis, long non coding RNA IFNG-AS1. Mol Biol Rep. 2019;46(6):6495-500.

19. Sansanayudh N, Numthavaj P, Muntham D, Yamwong S, McEvoy M, Attia J, et al. Prognostic effect of mean platelet volume in patients with coronary artery disease. J Thromb Haemost. 2015;114(12):1299309 .

20. Nozawa N, Hibi K, Endo M, Sugano T, Ebina T, Kosuge M, et al. Association between circulating monocytes and coronary plaque progression in patients with acute myocardial infarction. Circulation. 2010;74(7):1384-91.

21. Hafiane A, Genest J. High density lipoproteins: measurement techniques and potential biomarkers of cardiovascular risk. BBA Clin. 2015;31(3):175-88.

22. Sansanayudh N, Anothaisintawee T, Muntham D, McEvoy M, Attia J. Mean platelet volume and coronary artery disease: a systematic review and meta-analysis. Int J Cardiol. 2014;175(3):433-40.

23. McManus D, Freedman J. MicroRNAs in platelet function and cardiovascular disease. Nat Rev Cardiol. 2015;12(12):711-7.

24. Zhou D, Wang G, Fan Y, Wan Z, Liu X. Platelet to lymphocyte ratio is associated with the severity of coronary artery disease and clinical outcomes of percutaneous coronary intervention in the Chinese Han population. Exp Ther Med. 2017;13(2):731-8.

25. Hartwig H, Roig CS, Daemen M, Lutgens E, Soehnlein O. Neutrophils in atherosclerosis. A brief overview. J Hamostaseologie. 2015;35(2):121-7.

26. Soehnlein O. Multiple roles for neutrophils in atherosclerosis. Circ Res. 2012;110(6):875-88.

27. Kalay N, Dogdu O, Koc F, Yarlioglues M, Ardic I, Akpek M, et al. Hematologic parameters and angiographic progression of coronary atherosclerosis. Angiology. 2012;63(3):213-7.

28. Nilsson L, Wieringa WG, Pundziute G, Gjerde M, Engvall J, Swahn E, et al. Neutrophil/Lymphocyte ratio is associated with non-calcified plaque burden in patients with coronary artery disease. PloS One. 2014;9(9):e108183.

29. Chen J, Chen MH, Li S, Guo YL, Zhu CG, Xu RX, et al. Usefulness of the neutrophil-to-lymphocyte ratio in predicting the severity of coronary artery disease: a Gensini score assessment. J Atheroscleros Thrombos. 2014;21(12):1271-82.

30. Zhang GY, Chen M, Yu ZM, Wang XD, Wang ZQ. Relation between neutrophil-to-lymphocyte ratio and severity of coronary artery stenosis. Gene Mol Res. 2014;13(4):9382-9. 